Remarquons cependant que la Hollandaise, comme génisse, dépasse la Flamande, tandis que la Brabançonne, qui occupe le $3^{\mathrm{e}}$ rang comme génisse, est refoulée au $6^{\mathrm{e}}$ rang après le $4^{\mathrm{e}}$ veau. La Dairy-Shorthorn, ainsi que les produits issus du croisement de celle-ci avee la Rouge des Flandres n'a donné que des sujets de valeur secondaire. Enfin la race allemande, restant des fournitures faites en réparation des dommages de guerre, occupe la dernière place aussi bien comme génisse que comme vache de $4^{\mathrm{e}}$.veau.

En présence de résultats aussi concluants, les éleveurs de la région Gheluwe-Wervicq n'hésiteront plus à maintenir les races du Pays. II est incontestable que l'introduction du bétail anglais, sous forme de dons et sous forme d'achats, ainsi que la répartition des bovins récupérés en Allemagne, ont rendu les plus grands services et ont permis la reconstitution extraordinairement rapide de l'agriculture dans la région dévastée. Mais à présent que l'exploitation agricole est redevenue normale et que l'élevage peut être pratiqué à nouveau avec l'unique souci de l'amélioration du cheptel, les membres du Syndicat de Gheluwe-Wervicq, s'appliqueront à l'épuration progressive de leurs étables en ne conservant que les races autochtones et dans celles-ci les lignées les plus intéressantes.

\title{
NOUVELLE MÉTHODE DE MICRODOSAGE MOLYBDOMANGANIMÉTRIQUE DU LACTOSE
}

\author{
par Georges FONTÊS et Lucien THIVOLLE
}

Nous avons récemment publié (1) une méthode qui permet de doser, avec une approximation de 1 à $2 \%$, des quantités de glucose inférieures au milligramme et même au dixième de milligramme.

Cette méthode est rapide, facile, et se prête aux dosages en série. Il nous a paru utile de l'employer au micro-dosage du lactose.

\section{Principes de LA MÉTHOde}

On trouvera dans notre précédent travail (loc. cit) l'étude critique des différentes méthodes de dosage des sucres réducteurs et les raisons qui nous ont fait garder la liqueur cupro-tartrique en milieu alcalin. Mais nous avons beaucoup amélioré les solutions classiques. La liqueur dont la formule est donnée ci-après possède les avantages suivants :

a) Elle ne donne pas d'auto-réduction.

b) Elle permet d'obtenir un sous-oxyde de cuivre microchimique aussi inoxydable que celui produit par de grandes quantités de sucres réducteurs et qui est, de plus, très aisé à filtrer et de dissolution facile.

(1) G. Fontes et L. Thrvoule : Recherches expérimentales sur le microdosage des substances glucidiques réductrices du sang. (Bulletin de la Société de Chimie Biologique, t. IX, pp. 353-445 1927). 
c) Elle ne détruit pas de sucre. II s'ensuit, l'auto-réduction étant nulle, que les quantités de sous-oxyde obtenues sont rigoureusement proportionnelles aux quantités de sucre mises en œuvre.

La méthode consiste donc, dans un premier temps, à produire du sous-oxyde par l'action du lactose, à chaud, sur une liqueur cuprotartrique alcaline.

$\mathrm{Ce} \mathrm{Cu}^{2} \mathrm{O}$ est ensuite séparé par filtration des autres produits de la réaction. Cette opération est rendue nécessaire par la présence d'un composé réducteur, issu de l'acide tartrique et qui gêneraitle virage final.

Dans un dernier temps, ce sous-oxyde est dosé par molybdomanganimétrie.

Rappelons les faits essentiels sur lesquels repose cette méthode générale d'analyse microchimique:

Lorsqu'un corps, susceptible de fixer de l'oxygène, est mis en présence d'une solution incolore d'anhydride molybdique dans l'acide phosphorique, il passe à son maximum d'oxydation en empruntant son oxygène à $\mathrm{MoO}^{3}$. Ce dernier est done réduit et se transforme en sous-oxyde de molybdène intensément coloré en bleu.

Cette première phase peut être schématisée par l'équation :

$$
\mathrm{MoO}^{3}-\mathrm{O}=\mathrm{MoO}^{2}
$$

Ce bleu de molybdène résiste pendant des heures à l'action de l'air. Par contre il est oxydé instantanément et régulièrement par une solution très diluée de permanganate de potassium. Ici, comme en manganimétrie ordinaire, $2 \mathrm{MnO}^{4} \mathrm{~K}$ fournissent 50 .

La fin de la réaction a lieu lorsqu'une goutte de $\mathrm{MnO}^{4} \mathrm{~K}$ provoque une décoloration brusque de la solution.

Dans le cas du dosage d'un sucre réducteur, les quantités de permanganate ainsi versées sont rigoureusement proportionnelles au $\mathrm{Cu}^{2} \mathrm{O}$ obtenu done au sucre mis en œuvre.

\section{RÉACTIFs}

\section{Liqueur cupro-tartrique alcaline.}

Solution A. - Dissoudre à l'ébullition $50 \mathrm{gr}$. de tartrate acide de potassium très pur, exactement pesés, dans $800 \mathrm{~cm}^{3}$ d'acide sulfurique normal. Faire d'autre part une solution de $25 \mathrm{gr}$. de sulfate de cuivre cristallisé, exactement pesés, dans environ $80 \mathrm{~cm}^{3}$ d'eau et l'ajouter à la précédente. Refroidir. Compléter à $1.000 \mathrm{~cm}^{3}$ avec de l'eau distillée. La solution doit être limpide. Filtrer si c'est nécessaire en utilisant le tube filtrant ci-dessous décrit.

Une telle solution ne présente, au moment de sa préparation, aucune auto-réduction et garde cet avantage pendant plusieurs semaines.

Solution $B$. - Sulfate de potasse eristallisé pur à 80 gr. par litre (1).

(1) Cette solution a pour but de donner un sous-oxyde floculé aussi inoxydable que celui que l'on obtient en macro-chimie. 
Solution C. - Carbonate de potasse pur et sec à 280 gr. par litre.

La liqueur définitive s'obtient en mélangeant au moment de l'emploi un volume, exactement mesuré , de chaque solution dans l'ordre $A, B, C$. Ce mélange doit se faire dans un vase propre et ne contenant notamment plus trace d'une liqueur antérieure.. Enfin faire toujours une minime quantité du mélange juste suffisante pour les besoins immédiats. Grâce à ces petites précautions, l'auto-réduction est nulle ou tout à fait négligeable et le sous-oxyde est obtenu dans l'état optimum pour sa filtration et sa dissolution futures.

\section{Réactif molybdique phosphọrique.}

Prendre dans une capsule de porcelaine $20 \mathrm{gr}$. d'acide molybdique pur ou $40 \mathrm{gr}$. de molybdate d'ammoniaque pur. Ajouter environ $300 \mathrm{~cm}^{3}$ d'eau et $10 \mathrm{gr}$. de soude en bâtons (acide molybdique) ou $20 \mathrm{gr}$. (molybdate). Faire bouillir jusqu'à complet départ d'ammoniaque. Rajouter environ $500 \mathrm{~cm}^{3}$ d'eau et $200 \mathrm{gr}$. d'acide phosphorique cristallisé pur en solution dans au moins $200 \mathrm{~cm}^{3}$ d'eau. Faire bouillir un quart d'heure. Ajouter pendant l'ébullition quelques gouttes d'une solution concentrée de permanganate de potasse jusqu'à persistance de coloration rosée. Refroidir. Verser goutte à goutte une solution diluée de sulfate ferreux jusqu'à déeoloration complète. Compléter au litre.

3. Solution de permanganate de potassium à $0 \mathrm{gr} .08$ par litre.

Faire une solution stock à $8 \mathrm{gr}$. par litre qui, en quelques jours, prend et conserve un titre invariable. Diluer 100 fois au moment de l'usage. Cette dilution se conserve plusieurs semaines sans baisser de titre. 4. Solution de lactose pur et sec à un pour 5.000 .

Conservée sous toluène ou sous xylol, cette solution est inaltérable.

\section{DOSAGE DU LACTOSE EN SOLUTION PURE}

\section{Description de la technique.}

Choisir des tubes à essais de $20 \mathrm{~cm}$. de haut sur $2 \mathrm{~cm}$. de diamètre. Les laver au mélange sulfo-chromique puis à l'eau distillée. Les faire sècher.

Verser dans chacun d'eux, au moyen d'une micro-burette, des quantités soigneusement mesurées d'une solution de lactose à $1 \mathrm{gr}$. par litre. Compléter. le volume à $5 \mathrm{~cm}^{3}$ avec de l'eau distillée et ajouter $2 \mathrm{~cm}^{3}$ de la,liqueur cupro-tartrique earbonatée préparée selon les indications ci-dessus. Ces $2 \mathrm{~cm}^{3}$ doivent être versés de manière à entraîner au fond du tube les moindres traces de solution lactosée qui pourraient adhérer à ses parois.

Porter les tubes (en nombre aussi grand qu'on le désirera) dans un bain d'eau en complète ébullition et les y laisser vingt minutes. Ce temps 
est nécessaire pour permettre au sous-oxyde de subir l'agglomération optima.

Refroidir les tubes sous un courant d'eau ou attendre leur refroidissement spontané.

Filtrer le sous-oxyde au moyen de l'appareil dont on trouvera cicontre un dessin (Fig. I) et dont voici la description :

Un tube de verre $\mathrm{F}$ évasé à sà partie supérieure et étiré à sa partie inférieure présente, près de celle-ci, un petit morceau de toile de platine à mailles fines $(0,2 \mathrm{~mm}$ environ) soudé au chalumeau dans l'épaisseur même du verre. (Ch. VoLck, constructeur à Strasbourg). Ce tube est enfoncé par sa partie étirée dans un bouchon de caoutchouc et l'ensemble est monté sur une fiole à vide $\left(V_{1}\right)$. Celle-ci est reliée à une seconde fiole analogue $\left(V_{2}\right)$ par du caoutchouc à vide et par l'intermédiaire d'un tube en verre fort coudé, enfoncé dans un bou-

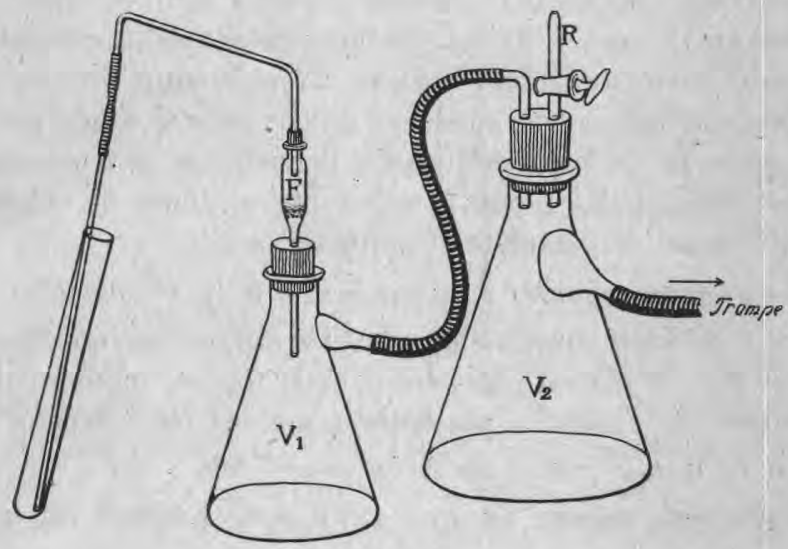

Fig. 1

chon en caoutchouc à deux trous. L'autre trou porte un robinet $(R)$ dont l'ouverture réglable permet une plus ou moins grande entrée d'air dans l'ensemble de l'appareil. On règle ainsi à volonté la vitesse de la filtration. L'ensemble de l'appareil est mis en communication avec une trompe à eau.

Garnir d'amiante la toile de platine. Se servir d'amiante en fibres dures susceptibles d'être finement broyée au mortier avec un peu d'eau, Après broyage laver l'amiante à l'acide chlorhydrique bouillant, puis à plusieurs reprises à l'eau distillée jusqu'à disparition de toute réaction acide. Conserver le produit ainsi préparé dans de l'eau distillée.

Au moment de la préparation du tube filtrant, mettre l'amiante en suspension et en verser une certaine quantité dans l'appareil sans faire le vide. Etablir ensuite graduellement l'aspiration de manière 
que l'amiante se tasse sans lacune sur la toile de platine. Déposer ainsi une couche d'amiante d'environ $1 \mathrm{~cm}$. d'épaisseur.

Boucher alors la partie supérieure du tube par un petit bouchon de caoutchoue portant un tube en verre deux fois coudé à angle droit, d'un diamètre intérieur de $3 \mathrm{~mm}$. et dent l'extrémité de la branche des. cendante, étirée en pointe, plonge au fond du tube où a eu lieu la réduction. On rend cette partie de l'appareil beaucoup plus maniable en coupant la partie descendante du tube un peu au-dessus du niveau où il affleure l'orifice du tube de réduction et en raccordant les deux fragments avec un petit morceau de caoutchouc. Avant de procéder à une filtration, laver le tube coudé et l'amiante par aspiration d'environ $20 \mathrm{~cm}^{3}$ d'eau distillée.

Pour filtrer du sous-oxyde, ouvrir $R$ en totalité et introduire la pointe effilée au fond du tube à réduction. Puis fermer très légèrement $\mathrm{R}$, de manière à réaliser une aspiration de deux gouttes par seconde. jusqu'à ce que la totalité du liquide contenu dans le tube ait été aspirée. A ce moment là (une partie ou la totalité du $\mathrm{Cu}^{2} \mathrm{O}$ se trouvant sur l'amiante) fermer $R$ en totalité et maintenir ce violent courant d'air au moins 30 secondes de manière à obtenir du sous-oxyde bien séparé de la liqueur dans laquelle il a pris naissance. Se contenter de ce "lavage par l'air " et ne jamais laver à l'eau. Cette dernière pratique aurait pour conséquence de remettre $\mathrm{Cu}^{2} \mathrm{O}$ en suspension fine et de le rendre oxydable.

La filtration étant ainsi terminée, adapter le tube filtrant (toujours en relation par l'intermédiaire du tube deux fois coudé avec le tube à réduction) sur une autre fiole à vide. Verser dans le tube à réduction quelques $\mathrm{cm}^{3} \mathrm{du}$ réactif molybdique et agiter pour aider la dissolution du sous-oxyde présent. Puis relier l'ensemble de l'appareil à V2 et établir une aspiration modérée. Le réactif tombe sur le sous-oxyde rassemblé sur l'amiante et le dissout en donnant du bleu de molybdène. On favorise cette dissolution en supprimant quelques instants l'aspiration. Répéter l'opération jusqu'à ce que le réactif molybdique ne présente plus la moindre trace de coloration bleue après avoir traversé la couche d'amiante.

Laver alors l'ensemble du tube à réduction et de l'appareil filtrant avee de l'eau distillée sans oublier le bouchon qui ferme le tube à amiante. A la suite de cette opération le tube filtrant est prêt pour une nouvelle récolte de sous-oxyde et le bleu de molybdène provenant du dosage en cours se trouve quantitativement dans la fiole à vide.

Il ne reste plus qu'à doser ce bleu. Pour cela, transvaser le contenu de la fiole à vide dans une capsule de porcelaine et rincer la fiole à l'eau distillée jusqu'à complète absence de coloration.

Puis, d'une burette graduée, faire tomber goutte à goutte la solution diluée de permanganate, jusqu'à décoloration complète. Sur le 'fond 
blanc de la capsule, ce virage s'observe toujours très aisément en lumière naturelle ou artificielle, et il est produit par une unique goutte $\mathrm{du}$ permanganate très dilué (environ $\mathrm{N} / 400$ ) que l'on emploie.

\section{Résultats obtenus.}

Le tableau ci-après démontre qu'il est possible de doser, grâce à notre méthode des quantités de lactose comprises entre 0 et 2,5 $\mathrm{mgr}$. avec une approximation excellente. Ces limites sont suffisantes pour permettre l'étude de tous les laits. On peut constater la proportionnalité qui se manifeste entre tous nos chiffres rapportés au milligramme. Portés en courbe, ils donnent une droite parfaite passant par le zéro des coordonnées. Ces résultats sont obtenus quelque soit le nombre de tubes simultanément utilisés et sans qu'il soit nécessaire d'avoir un témoin pour chaque série. De plus nous utilisons seulement un bain d'eau et non plus un bain de chlorure de calcium et nous avons supprimé la centrifugation. On voit done que la méthode actuelle présente des avantages sur celle que nous avons précédemment décrite (1).

\begin{tabular}{|c|c|c|c|c|c|}
\hline $\begin{array}{l}\text { Lactose } \\
\text { utilisé } \\
\text { (mgr) }\end{array}$ & $\begin{array}{r}\text { Perm } \\
\mathrm{v} \\
\text { (c }\end{array}$ & $\begin{array}{l}\text { langanate } \\
\text { versé } \\
\left(\mathrm{cm}^{3}\right)\end{array}$ & $\begin{array}{l}\text { Moyenne des } \\
\text { chiffires } \\
\text { précédents }\end{array}$ & $\begin{array}{l}\text { Valeur calculée } \\
\text { pour } 1 \mathrm{mgr} \text {. } \\
=9 \mathrm{~cm}^{3}\end{array}$ & $\begin{array}{l}\text { Erreur } \\
\text { p. } 100\end{array}$ \\
\hline 0,2 & 1,80 & $-\quad 1,80$ & 1,80 & 1,80 & 0 \\
\hline 0,3 & 2,70 & $-\quad 2,60$ & 2,65 & 2,70 & $-1,85$ \\
\hline 0,4 & 3,70 & $-\quad 3,60$ & 3,65 & 3,60 & $+1,38$ \\
\hline 0,5 & 4,5 & $-4,5$ & 4,5 & 4,5 & 0 \\
\hline 0,6 & 5,30 & $-\quad 5,40$ & 5,35 & 5,40 & $-0,92$ \\
\hline 0,7 & 6,30 & $-6,30$ & 6,30 & $6 ; 30$ & 0 \\
\hline 0,8 & 7,10 & $-7,10$ & 7,10 & 7,20 & $-1,38$ \\
\hline 0,9 & $8, \cap 0$ & $-8,10$ & 8,05 & 8,10 & $-0,62$ \\
\hline 1,0 & 9,0 & $-9,0$ & 9,0 & 9,0 & \\
\hline 1,1 & 9,80 & $-10,0$ & 9,90 & 9,90 & 0 \\
\hline 1,2 & 10,7 & $-10,8$ & 10,75 & 10,8 & $-0,45$ \\
\hline 1,3 & 11,7 & $-11,7$ & 11,7 & 11,7 & 0 \\
\hline 1,4 & 12,5 & $-12,7$ & 12,6 & 12,6 & 0 \\
\hline 1,5 & 13,5 & $-13,6$ & 13,55 & 13,5 & $+0,37$ \\
\hline 1,6 & 14,3 & $-14,3$ & 14,3 & 14,4 & -0, \\
\hline 1,7 & 15,2 & $-15,4$ & $i 5,3$ & 15,3 & 0 \\
\hline 1,8 & 16,4 & $-16,4$ & 16,4 & 16,2 & $+1,22$ \\
\hline 1,9 & 17,1 & $-17,1$ & 17,1 & 17,1 & 0 \\
\hline 2,0 & 18,1 & $-18,1$ & 18,1 & 18,0 & $+0,55$ \\
\hline 2,1 & 19,0 & $-19,0$ & 19,0 & 18,9 & $+0,53$ \\
\hline 2.2 & 19,7 & $-19,7$ & 19,7 & 19,8 & $-0,5$ \\
\hline 2,3 & $-20,6$ & $-20,6$ & 20,6 & 20,7 & $-0,47$ \\
\hline 2,4 & 21,6 & $-21,6$ & 21,6 & 21,6 & 0 \\
\hline 2,5 & 22,5 & $-22,5$ & 22,5 & 22,5 & 0 \\
\hline
\end{tabular}

(1) G. Fontes et L. Thrvoune. Méthode de microdosage manganimétrique du lactose. Application au laiv :


pp. 164.165 (suite et fin) 


\section{DOSAGE DU LACTOSE DANS LE LAIT}

1. Mensuration du lait. Le dosage peut aussi bien s'effectuer à partir de $1 \mathrm{~cm}^{3}$ de lait qu'à partir de $0 \mathrm{~cm}^{3} 1$ ou de $0 \mathrm{~cm}^{3} 05$.

Dans tous les cas on devra utiliser une pipette à écoulement total graduée au mercure. Pour que la mesure soit correcte, il est nécessaire de tremper le moins possible la pipette dans le lait à mesurer et de ne dépasser qu'au minimum le trait de jauge supérieur, au-moment de l'aspiration. En outre, la mesure terminée, tenir la pipette bouchée par l'index, la pointe en l'air et essuyer cette pointe avec un morceau de papier buvard.

2. Dilution du lait. Commme le montrent les chiffres ci-après, on obtient des résultats identiques en opérant sur le même lait, si la dilution finale varie du simple au double. L'essentiel est d'avoir dans la prise du filtrat final moins de 2,5 mgr. de lactose. Mais, eomme on diminue l'erreur relative de la mensuration quand les quantités mesurées augmentent, il est préférable de faire subir au lait une dilution de 1/200 et d'opérer sur $5 \mathrm{~cm}^{3} \mathrm{du}$ filtrat final.

Pour $1 \mathrm{~cm}^{3}$ de lait il sera done utile d'avoir une fiole jaugée de $200 \mathrm{~cm}^{3}$. (A défaut on prendra un matras de $100 \mathrm{~cm}^{3}$ et on utilisera $2 \mathrm{~cm}^{3}$ du dernier filtrat auxquels on ajoutera $3 \mathrm{~cm}^{3}$ d'eau distillée.)

Pour $0,1 \mathrm{~cm}^{3}$ une fiole de $20 \mathrm{~cm}^{3}$ convient parfaitement. Pour $0,05 \mathrm{~cm}^{3}$ on se servira d'une fiole de $10 \mathrm{~cm}^{3}$.

Le lait, mesuré comme il a été dit plus haut, est introduit dans le récipient choisi. Souffler dans la pipette, puis la rincer à plusieurs reprises à l'eau distillée, de manière à entraîner les moindres traces de lait adhérentes par viscosité. Il n'y a aucun inconvénient à utiliser pour ce lavage un volume d'eau égal à la moitié environ de la capacité du vase jaugé.

3. Défécation. Au volume de lait ainsi introduit dans le matras, ajouter un égal volume de réactif de BIERRY et MOQUET dont voici la formule :

A $400 \mathrm{gr}$. de nitrate mercurique, ajouter environ $700 \mathrm{~cm}^{3}$ d'eau. Chauffer vers $40^{\circ}$. Verser une quantité d'acide nitrique pur suffisante pour tout dissoudre puis, goutte à goutte, de la lessive de soude jusqu'à précipité persistant. Compléter à $1.000 \mathrm{~cm}^{3}$ avec de l'eau distillée. Filtrer:

L'introduction de ce réactif doit se faire goutte à goutte, en remuant sans cesse, de manière à éviter la formation de gros grumeaux de défécat.

Ajouter ensuite quelques gouttes d'une solution aleoolique de phénol-phtaléine, puis de la soude ou de la potasse environ normale jusqu'à coloration rouge persistante. Enfin revenir en milieu légèrement 
acide (environ $\mathrm{Ph}=6,5)$ par l'adjonction d'une solution d'acide acétique à $5 \%$ dont on ménagera un excès de deux gouttes.

Ajouter de l'eau jusqu'au trait de jauge. Mélanger. La défécation est terminée.

4. Démercuration. Placer dans un flacon bouché à l'émeri environ 200 mgr. de poudre de cuivre (le cuivre pur réduit Poulenc eonvient parfaitement) et filtrer directement sur lui le produit des opérations précédentes. Dans le cas de $1 \mathrm{~cm}^{3}$ de lait recueillir seulement environ 20 $\mathrm{cm}^{3}$ de filtrat. Fermer le flacon et agiter vigoureusement pendant une minute. Laisser reposer une dizaine de minutes et filtrer à nouveau, de façon à éliminer les moindres parcelles de cuivre. On obtient ainsi un liquide qui ne renferme plus trace de mercure et qui est prêt pour le dosage (1).

5. Dosage. On se retrouve dans le eas d'une solution pure de lactose. Mesurer $5 \mathrm{~cm}^{3} \mathrm{du}$ filtrat final (ou une quantité moindre si l'on craint d'avoir plus de 2,5 mgr. de lactose, que l'on portera à $5 \mathrm{~cm}^{3}$ par adjonction d'eau distillée). Ajouter $2 \mathrm{~cm}^{3}$ de la liqueur cupro-tartrique alcaline. Laisser 20 minutes au bain-marie bouillant. Filtrer le sous-oxyde, le dissoudre dans le réactif molybdique et doser le bleu de molybdène produit au permanganate dilué. Soit $\mathrm{N}$ le nombre de $\mathrm{cm}^{3}$ de $\mathrm{MnO}^{4} \mathrm{~K}$ ainsi versé.

6. Calcul du dosage. On aura préalablement titré le permanganate au moyen de la solution étalon de lactose à 5 pour 1.000 dont $5 \mathrm{~cm}^{3}$ renferment $1 \mathrm{mgr}$. Soit $\mathrm{T}$ le nombre de $\mathrm{cm}^{3}$ de permanganate qui représente $1 \mathrm{mgr}$. de lactose.

La quantité de lactose dans les filtràts utilisés sera donnée en mgr. par le rapport $\mathrm{N} / \mathrm{T}$. II suffira de rapporter ee nombre au volume total de la dilution pour avoir la quantité de lactose dans la prise effectuée et par suite par litre de lait.

7. Justification de la méthode. Les chiffres ci-dessous ont été obtenus à partir du même échantillon de lait de vache (1 mgr. de lactose $=9,6 \mathrm{~cm}^{3}$ de permanganate). Comme on le voit, quelle que soit la. quantité de lait mesurée ou la dilution effectuée, on trouve des chiffres dont les plus divergents $(46,4$ et 47,48$)$ présentent entre eux une erreur de $2 \%$. La moyenne des 8 chiffres ainsi obtenus $(46,8)$ est considérée comme le chiffre exact du taux du lactose. Par rapport à lui, du lactose rajouté au même échantillon de lait se retrouve avec une approximation excellente.

Signalons que tous ces dosages ont fait l'objet d'une seule série de manipulations.

(1) Pour la justification de cette partie de la technique prière de se rapporter à notre mémoire sur le dosage de "glucosè "sanguin (loc. cit.). 


\begin{tabular}{|c|c|c|c|c|c|c|c|c|}
\hline \multirow{2}{*}{$\begin{array}{l}\text { Lait } \\
\text { mesu- } \\
\text { ré } \\
\left(\mathrm{mm}^{3}\right)\end{array}$} & $\begin{array}{l}\text { Lactose } \\
\text { ajouté } \\
\text { (mgr) }\end{array}$ & $\begin{array}{l}\text { Dilu- } \\
\text { tion } \\
\text { finale } \\
\left(\mathrm{cm}^{3}\right.\end{array}$ & $\begin{array}{l}\text { Filtrat } \\
\text { utilisé } \\
\left(\mathrm{cm}^{3}\right)\end{array}$ & $\begin{array}{l}\text { Perman- } \\
\text { ganate } \\
\text { yersé } \\
\left(\mathrm{cm}^{3}\right)\end{array}$ & $\begin{array}{l}\text { Lactose } \\
\text { dans le fil- } \\
\text { trat utilisé } \\
\text { (mgr) }\end{array}$ & $\begin{array}{l}\text { Lactose } \\
\text { trouvé } \\
\text { (mgr) }\end{array}$ & $\begin{array}{l}\text { Lactose qu'on } \\
\text { devait trouver } \\
\text { (mgr) }\end{array}$ & $\begin{array}{l}\text { Erreur } \\
\text { p. } 100\end{array}$ \\
\hline & - & & - & & & - & $\rightarrow$ & - \\
\hline \multirow{2}{*}{1} & \multirow{2}{*}{0} & \multirow{2}{*}{200} & 5 & 11,2 & 1,16 & 46,4 & & \\
\hline & & & 5 & 11,2 & 1,16 & 46,4 & & \\
\hline \multirow{2}{*}{1} & \multirow{2}{*}{0} & \multirow{2}{*}{100} & 2 & 8,9 & 0,93 & 46,5 & & \\
\hline & & & 2 & 9,0 & 0,936 & 46,8 & & . \\
\hline \multirow{2}{*}{0,1} & \multirow{2}{*}{0} & \multirow{2}{*}{20} & 15 & 11,3 & 1,178 & 47,12 & . & \\
\hline & & & 5 & 11,4 & 1,187 & 47,48 & . & \\
\hline \multirow[t]{2}{*}{0,05} & \multirow[t]{2}{*}{0} & \multirow{2}{*}{10} & $\{5$ & 11,2 & 1,16 & 46,4 & & - \\
\hline & & & 1,9 & 4,3 & 0,448 & 47,1 & & \\
\hline & \multirow[t]{2}{*}{25} & \multirow[t]{2}{*}{250} & $\left\{\begin{array}{l}5 \\
5\end{array}\right.$ & 13,85 & 1,44 & 72,0 & $46,8+25=71,8$ & $+0,28$ \\
\hline & & & 5 & 13,85 & 1,44 & 72,0 & & \\
\hline & 10 & 200 & 5 & 13,1 & 1,40 & 56,0 & $46,8+10=56,8$ & $-1,4$ \\
\hline
\end{tabular}

(Institut de Chimie Biologique de la Faculté de Médecine de Strasbourg.)

\title{
BIBLIOGRAPHIT ANALYTIQUE
}

\author{
10 LES LIVRES
}

\section{Waksman (S. A.) et Davison (W. C.). - Enzymes - Properties -} Distribution - Methods and Applications (Enzymes - Propriétés - Distribution - Recherches et Applications). 1 vol. relié, 364 p. The Williams et Wilkins Company, Ed. Baltimore, 1926. Prix: 5 dollars.

Voilà un livre nouveau, paru en septembre dernier, dans lequel les auteurs se sont employés à rassembler dans une forme aussi concise que possible toutes les informations importantes nécessaires à connaître sur les enzymes.

Leur travail se termine par une bibliographie particulièrement soignée, avec près de 1.400 références, car il en est plusieurs qui s'abritent sous le même numéro, de façon que le chercheur trouve ainsi les sources des documents originaux qui lui sont nécessaires s'il veut entrer dans le détail de l'étude de certains des ferments solubles, dont W. et D. parlent dans leur ouvrage.

Il faut rendre hommage à la conscience avec laquelle ils ont rédigé celui-ci. Sans doute, se sont-ils trouvés devant les mêmes difficultés que rencontrent ceux qui, pour la première fois, abordent l'étude des enzymes, difficultés tenant à l'aceumulation des documents sur le sujet, accumulation qui ne va pas, évidemment, sans d'apparentes contradictions dans beaucoup des résultats signalés; aussi ont-ils fait pour eux d'abord ce que nous pouvons juger qu'ils ont fait pour nous maintenant, c'est-à-dire le dépouillement, la plupart du temps dans le document original, de près de 2.000 travaux.

Après avoir parlé de l'importance des enzymes dans les processus biologiques, ils abordent la chimie des ferments et les réactions qu'ils déterminent, puis l'étude des facteurs quị influencent celles-ci. Ensuite vient la distribution des enzymes. Ils réservent la part la plus grande aux enzymes du corps humain ou des animaux, le chapitre des enzymes végétales est sobrement traité, un peu moins peutêtre celui des enzymes des micra-organismes. L'examen des méthodes pour la préparation et l'étude des enzymes vient après, ainsi que leur elassification. Enfin 